\title{
ON A CHARACTERIZATION OF $W$-SETS AND THE DIMENSION OF HYPERSPACES
}

\author{
J. GRISPOLAKIS AND E. D. TYMCHATYN
}

\begin{abstract}
A subcontinuum $A$ of a continuum $X$ is a $W$-set if for each mapping $f: Y \rightarrow X$ of an arbitrary continuum $Y$ onto $X$ there is a continuum in $Y$ which is mapped by $f$ onto $A$. We characterize $W$-sets in terms of accessibility by small continua. We localize several known results on continua all of whose subcontinua are $W$-sets. Finally, we extend a result of J. T. Rogers by proving that if $X$ is an atriodic continuum whose first Cech cohomology group is finitely generated then the hyperspace $C(X)$ of subcontinua of $X$ is two dimensional.
\end{abstract}

1. Introduction. By a continuum we mean a compact connected, metric space and by a mapping we mean a continuous function. A subcontinuum $K$ of a continuum $Y$ is said to be a $W$-set in $Y$ provided that for every mapping $f: Y \rightarrow Y$ (where $X$ is any continuum) onto $Y$ there exists a subcontinuum $L$ of $X$ which is mapped by $f$ onto $K$. A continuum $Y$ is said to belong to Class $(W)$ provided that every subcontinuum of $Y$ is a $W$-set. In [3-5] there were given some necessary and some sufficient conditions for a continuum to be in Class $(W)$. In $\S 2$ we give several analogous characterizations of $W$-sets.

By $H^{n}(X)$ we denote the $n$th Čech cohomology group with integer coefficients of the space $X$. A continuum $X$ is said to be a triod if there exists a subcontinuum $M$ of $X$ such that $X \backslash M$ has at least three components. If a continuum does not contain any triod it is said to be atriodic.

If $A$ is a subset of a space $X$ we let $\mathrm{Cl}(A)$ and $\operatorname{Bd}(A)$ denote the closure and boundary, respectively, of $A$ in $X$. For $\varepsilon>0$ we let $S(A, \varepsilon)$ denote the open $\varepsilon$-ball about $A$ with respect to a fixed but arbitrary metric on $X$.

If $X$ is a continuum we let $C(X)$ denote the hyperspace of subcontinua of $X$. We let $\mu: C(X) \rightarrow[0,1]$ denote a Whitney map from $C(X)$ onto [0,1] (i.e. if $A, B \in C(X)$ with $A \nsubseteq B$, then $\mu(A)<\mu(B)$ and $\mu(\{x\})=0$ for each $x \in X)$. The reader may consult $[\mathbf{1 1}]$ for properties of hyperspaces.

In $\S 3$ we prove that the property of being an atriodic cohomologically trivial continuum is inherited by all Whitney levels $\mu^{-1}(t)$ of the hyperspace. We also prove that if $X$ is an atriodic continuum with finitely generated $H^{1}(X)$, then $\operatorname{dim} C(X) \leq 2$. These results generalize results of Oversteegen and Tymchatyn [13] and Rogers [15].

Received by the editors November 2, 1984 and, in revised form, April 7, 1986.

1980 Mathematics Subject Classification (1985 Revision). Primary 54C10, 54B20; Secondary 54F 20, 54F 50 .

Key words and phrases. Continua, weakly confluent mappings, dimension of hyperspaces, atriodic.

The second author was supported in part by NSERC grant no. A5616

(C) 1987 American Mathematical Society $0002-9939 / 87 \$ 1.00+\$ .25$ per page 


\section{Some characterizations of $W$-sets.}

2.1 THEOREM. Let $A$ be a proper subcontinuum of a continuum $X$. Then $A$ is not $a W$-set in $X$ if and only if there exists some $\varepsilon>0$ and a neighborhood $G$ of $A$ such that

(1) for each $x \in G$ there exists a continuum $B$ from $x$ to $\operatorname{Bd}(G)$ such that $A \not \subset S(B, \varepsilon)$, and

(2) for each decomposition of $\operatorname{Bd}(G)=R \cup S$ into disjoint closed sets $R$ and $S$, there exists a continuum $K$ from $R$ to $S$ with $A \not \subset S(K, \varepsilon)$.

Proof. Suppose that $\varepsilon$ is a positive number and $G$ is a neighborhood of $A$ satisfying (1), (2), and $S(A, \varepsilon) \subset G$. Since $A$ is totally bounded, there exist an integer $n$ and a set $\left\{x_{1}, \ldots, x_{n}\right\} \subset A$ such that if $x \in G$ then there exists a continuum $B$ from $x$ to $\operatorname{Bd}(G)$ such that $B \cap S\left(x_{i}, \varepsilon / 2\right)=\varnothing$ for some $i \in\{1, \ldots, n\}$, and for each decomposition of $\operatorname{Bd}(G)$ into disjoint closed sets $R$ and $S$ there exists a continuum $B$ from $R$ to $S$ which misses $S\left(x_{i}, \varepsilon / 2\right)$ for some $i \in\{1, \ldots, n\}$.

For each $i \in\{1, \ldots, n\}$ let

$$
F_{i}=\left\{L \in C(X) \mid L \cap \operatorname{Bd}(G) \neq \varnothing \text { and } L \cap S\left(x_{i}, \varepsilon / 2\right)=\varnothing\right\} .
$$

For each $i, F_{i}$ is a compact subset of $C(X)$. Let $X_{i}=\bigcup_{i=1}^{n} F_{i}$. By [9, p. 23], $X_{i}$ is compact. By (1) and (2), we have that $X=\bigcup_{i=1}^{n} X_{i}$. Put $Y_{i}=X_{i} \times\{i\}$ and let $Z=\bigcup_{i=1}^{n} Y_{i}$. Let $f: Z \rightarrow X$ be the natural projection. Define an equivalence relation $\sim$ on $Z$ by setting $x \sim y$ if and only if either $x=y$ or $f(x)=f(y)$ and $f(x) \in X \backslash G$. Then $\sim$ is upper semicontinuous. Let $Y=Z / \sim$ be the quotient space and let $\phi: Z \rightarrow Y$ be the quotient map. Let $g: Y \rightarrow X$ be the unique mapping which causes the diagram

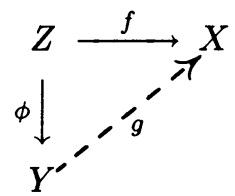

to commute. Since $f$ and $\phi$ are onto, $g$ is also onto. The set $g^{-1}(A)$ is the disjoint union of the closed subsets

$$
\phi\left[f^{-1}(A) \cap Y_{1}\right], \ldots, \phi\left[f^{-1}(A) \cap Y_{n}\right] .
$$

None of these sets maps onto $A$. Thus, no subcontinuum of $Y$ is mapped by $g$ onto $A$.

It remains to prove that $Y$ is a continuum. Clearly, each component of $Y$ meets $g^{-1}[\operatorname{Bd}(G)]$. By $(2)$ and the definition of $Y$, no decomposition of $g^{-1}[\operatorname{Bd}(G)]$ into disjoint closed sets extends to a decomposition of $Y$ into disjoint closed sets. Hence, $Y$ is connected. This completes the proof that $A$ is not a $W$-set in $X$.

Now, suppose that $A$ is not a $W$-set in $X$. Let $f: Z \rightarrow X$ be a mapping of a continuum $Z$ onto $X$ such that no subcontinuum of $Z$ is mapped by $f$ onto $A$. Let $U$ be an open neighborhood of $A$ such that no component of $f^{-1}[\mathrm{Cl}(U)]$ maps onto a set containing $A$. Let $\varepsilon>0$ so that $S(A, \varepsilon) \subset U$ and if $K$ is a component of $f^{-1}[\mathrm{Cl}(U)]$, then $f(K) \cap S(y, \varepsilon)=\varnothing$ for some $y \in A$. If $x \in U$ and $R$ is a component of $f^{-1}[\mathrm{Cl}(U)]$ which meets $f^{-1}(x)$, then $R \cap f^{-1}[\operatorname{Bd}(U)] \neq \varnothing$ by the Boundary Bumping Theorem (see $[11$, p. 625]). Hence, $f(R)$ is a continuum from $x$ to $\operatorname{Bd}(U)$ in $X$ such that $f(R) \cap S(y, \varepsilon)=\varnothing$ for some $y \in A$. So (1) holds for $U$. By 
taking an appropriate quotient space $Z$, we may suppose that $f$ maps $Z \backslash f^{-1}(U)$ homeomorphically onto $X \backslash U$.

Let $\operatorname{Bd}(U)=R \cup S$, where $R$ and $S$ are nonempty disjoint closed sets. Then $f^{-1}[\operatorname{Bd}(U)]=f^{-1}(R) \cup f^{-1}(S)$. If a component $K$ of $f^{-1}[\mathrm{Cl}(U)]$ meets both $f^{-1}(R)$ and $f^{-1}(S)$, then $f(K)$ is a continuum in $\mathrm{Cl}(U)$ from $R$ to $S$ so that $f(K) \cap S(y, \varepsilon)=\varnothing$ for some $y \in A$ by the choice of $U$ and $\varepsilon$. If no component of $f^{-1}[\mathrm{Cl}(U)]$ meets both $f^{-1}(R)$ and $f^{-1}(S)$, then some component $K$ of $f^{-1}(X \backslash U)$ meets both $f^{-1}(R)$ and $f^{-1}(S)$, since $Z$ is a continuum. Then $f(K)$ is a continuum in $X \backslash S(A, \varepsilon)$ from $R$ to $S$. So $U$ also satisfies (2). This completes the proof of the theorem.

From 2.1 we get the following intrinsic characterization of Class $(W)$.

2.2 Corollary. A continuum $X$ is in Class $(W)$ if and only if for every subcontinuum $A$ of $X$, for each $\varepsilon>0$ and each neighborhood $U$ of $A$ we have that either

(1) there exists $x \in U$ such that for every continuum $B$ from $x$ to $\mathrm{Bd}(U)$ in $\mathrm{Cl}(U)$ we have that $A \subset S(B, \varepsilon)$, or

(2) there exists a decomposition of $\operatorname{Bd}(U)=R \cup S$ into disjoint nonempty closed sets $R$ and $S$ such that for every subcontinuum $K$ of $X$ from $R$ to $S$ in $\operatorname{Cl}(U)$ we have that $A \subset S(K, \varepsilon)$.

The following is a local version of Theorem 3.2 in [5].

2.3 THEOREM. Let $X$ be a continuum and $A$ a subcontinuum of $X$. Then the following are equivalent:

(i) $A$ is a $W$-set in $X$,

(ii) for every Whitney map $\mu: C(X) \rightarrow[0, \infty)$ and for every subcontinuum $\Lambda$ of $\mu^{-1}(\mu(A))$ such that $\bigcup \Lambda=X$ we have $A \in \Lambda$,

(iii) if $X \subset Q$, the Hilbert cube, and $\left(Y_{i}\right)_{i=1}^{\infty}$ is a sequence of arcs in $Q$ such that $X=\lim Y_{i}$, then $A \in \lim \inf C\left(Y_{i}\right)$.

PROOF. The proof that (i) implies (ii) is very similar to the proof in $[\mathbf{5}$, Theorem 3.2] that (a) implies (b).

Suppose $A$ is a subcontinuum of $X$ satisfying (ii). Let $\left\{Y_{i}\right\}_{i=1}^{\infty}$ be a sequence of arcs in $Q$ such that $X=\lim _{i} Y_{i}$. Let $\Lambda$ be a Whitney level of $C(X)$ such that $A \in \Lambda$. By a slight modification of $[\mathbf{1 7}$, Theorem 1$]$ there is a Whitney map $\mu: C(Q) \rightarrow[0, \infty)$ such that $\Lambda \subset \mu^{-1}\left(t_{0}\right)$ for some $t_{0}$. Then $\Lambda$ is the Whitney level of $X$ with respect to $\mu \mid C(X)$. Let $\Lambda_{i}=\mu\left(t_{0}\right) \cap C\left(Y_{i}\right)$ for each $i$. Since $C(Q)$ is compact we may assume after passing to a subsequence if necessary that $\lim _{i} \Lambda_{i}=\Lambda^{\prime} \subset \Lambda$, where $\Lambda^{\prime}$ is a continuum. Then $\bigcup \Lambda^{\prime}=\lim _{i} \bigcup \Lambda_{i}=\lim _{i} Y_{i}=X$. Hence, $A \in \Lambda^{\prime} \subset \lim _{i} \inf C\left(Y_{i}\right)$. We have proved that (ii) implies (iii).

The proof that (iii) implies (i) is similar to the proof in [5, Theorem 3.2] that (c) implies (a).

3. Dimension of hyperspaces. It was proved by Kelley [9] that if $X$ is a hereditarily indecomposable continuum of dimension at least 2 , then $\operatorname{dim} C(X)=$ $\infty$. Bing proved that each continuum $X$ of dimension $\geq 3$ contains a hereditarily indecomposable continuum of dimension $\geq 2$. It remains an open question whether each continuum $X$ of dimension $\geq 2$ has $\operatorname{dim} C(X)=\infty$. It is well known that if 
$\operatorname{dim} C(X)<k$, then $X$ does not contain a $k$-od [11]. It would be very useful to know if Kelley's theorem is true for atriodic continua.

3.1 LEMmA. Let $X$ be an atriodic continuum, $x \in X$, and $\mu: C(X) \rightarrow[0,1]$ a Whitney map. Then $C_{t}(x)=\left\{K \in \mu^{-1}(t) \mid x \in K\right\}$ is either an arc or a point.

PrOOF. By Sorgenfrey [16, Theorem 1.8] if $A_{1}, A_{2}$, and $A_{3}$ are three continua in $X$ with a point in common, then $A_{1} \cup A_{2} \cup A_{3} \subset A_{i} \cup A_{j}$ for some $i, j \in\{1,2,3\}$.

Since $C_{t}(x)$ is compact it follows by a simple maximality argument that there exist $A, B \in C_{t}(x)$ such that $\bigcup C_{t}(x) \subset A \cup B$. If $A=B$ there is nothing to prove so we suppose $A \neq B$.

Suppose first that $A \cap B$ is connected and let $C \in C_{t}(x) \backslash\{A, B\}$. Then $C \subset$ $A \cup B$ and, since $A$ and $B$ are closed, $C \backslash A$ and $C \backslash B$ are separated sets. Since $A, B, C \in \mu^{-1}(t)$ and $\mu$ is a Whitney map, $C \not \subset B$ and $C \not \subset A$ so $C \backslash A$ and $C \backslash B$ are nonempty sets. If $A \cap B \not \subset C$, then $C \backslash(A \cap B)$ is connected by [3, Proposition 4.4] since $C \not \subset A \cap B$. This is a contradiction. Hence, $A \cap B \subset C$ for each $C \in C_{t}(x)$. If $C, D \in C_{t}(x)$ then either $C \backslash B \subset D$ or $D \backslash B \subset C$ by [16, Theorem 1.8] since $C \cup D \cup B$ does not contain a triod. Hence, the set $\left\{C \cap A \mid C \in C_{t}(x)\right\}$ is a chain in $C(X)$. Similarly, $\left\{C \cap B \mid C \in C_{t}(x)\right\}$ is a chain. It is well known that $C_{t}(x)$ is compact and arcwise connected. The lemma follows in this case.

If $A \cap B$ is not connected then $A \cap B$ consists of exactly two components $K$ and $L$ (by [3, Lemma 4.1]). We may choose $A$ and $B$ so that the component $K$ of $x$ in $A \cap B$ is minimal. By [3, Proposition 4.2] $A$ is a minimal continuum with respect to containing $K$ and $L$. In particular, if $R$ is a continuum in $A$ which meets both $K$ and $L$, then $A \backslash B \subset R$. If $C \in C_{t}(x) \backslash\{A, B\}$ and $C \cap L=\varnothing$, then $K$ separates $C$ and, hence, $K \subset C$ by [3, Proposition 4.4]. If $C \in C_{t}(x) \backslash\{A, B\}$ such that $C$ meets $L$, then $B \cup C=A \cup B$ and $C$ is unicoherent by [3, Proposition 4.2]. Since $C \not \subset A, K \cup L$ separates $C$. Since $C$ is unicoherent, either $K$ separates $C$ or $L$ separates $C$. If $K$ separates $C$, then $K \subset C$ by [3, Proposition 4.4]. If $K$ does not separate $C$, then $L$ separates $C$ and $L \subset C$ as above. Since $A \not \subset C$ is follows that $K \not \subset C$. Hence, $C$ and $B$ are continua such that $C \cup B=A \cup B$ and the component of $C \cap B$ which contains $x$ is a proper subset of $K$ which contradicts the minimality of $K$. Hence, if $C \in C_{t}(x)$, then $K \subset C$. The proof now proceeds as in the case where $A \cap B$ is connected.

3.2 THEOREM. If $X$ is an atriodic continuum with $\check{H}^{1}(X)=0$ and $\mu$ is a Whitney map for $X$, then for each $t \in[0,1], \mu^{-1}(t)$ is an atriodic one-dimensional continuum with $\check{H}^{1}\left(\mu^{-1}(t)\right)=0$.

PROOF. If $\Lambda$ is a subcontinuum of $\mu^{-1}(t)$, then $\bigcup \Lambda$ is a subcontinuum of $X$. By [6, Theorem 4.4] $X$ is one-dimensional and, hence, $\check{H}^{1}(\bigcup \Lambda)=0$. Also, $\bigcup \Lambda$ is atriodic and one-dimensional. By a theorem of Davis $[\mathbf{1}] \cup \Lambda$ is in Class $(W)$. By [5, Theorem 3.2] $\Lambda$ is a Whitney level of $\bigcup \Lambda$. By $[\mathbf{1 1}, 14.73 .3] \Lambda$ is an irreducible continuum. In particular, $\Lambda$ is not a triod. Hence, $\mu^{-1}(t)$ is atriodic.

To see that $\breve{H}^{1}\left(\mu^{-1}(t)\right)=0$ let

$$
K=\left\{(A, x) \mid x \in A \in \mu^{-1}(t)\right\} \subset \mu^{-1}(t) \times X .
$$

Let $\pi_{1}: K \rightarrow \mu^{-1}(t)$ and $\pi_{2}: k \rightarrow X$ be the coordinate projections. By Lemma 3.1 $\pi_{2}^{-1}(x)$ is an arc or a point for each $x \in X$. By the Hurewicz Theorem, $\operatorname{dim}(K) \leq 2$. 
Since $\check{H}^{1}(X)=0, \check{H}^{1}(K)=0$ by the Vietoris Mapping Theorem. Since $\pi_{1}$ is monotone, the homomorphism $\pi_{1}^{*}: \check{H}^{1}\left(\mu^{-1}(t)\right) \rightarrow \check{H}^{1}(K)$ is a monomorphism by [2]. Thus, $\check{H}^{1}\left(\mu^{-1}(t)\right)=0$. By [6] $\mu^{-1}(t)$ is one-dimensional.

3.3 Proposition. If $X$ is an atriodic continuum and $\check{H}^{1}(X)=0$, then $\operatorname{dim}(C(X)) \leq 2$.

PROOF. For each $t \in[0,1], \mu^{-1}(t)$ is a continuum of dimension no more than 1. The proposition follows from the Hurewicz Theorem.

Proposition 3.3 had been proved in [13] for tree-like continua. The theorem that follows generalizes Proposition 3.3. It also generalizes a result of Rogers [15] for hereditarily indecomposable continua.

3.4 THEOREM. If $X$ is an atriodic continuum such that $\check{H}^{1}(X)$ has finite rank, then $\operatorname{dim}(C(X)) \leq 2$.

Proof. By [6] $\operatorname{dim} X \leq 1$. Let $\mu: C(X) \rightarrow[0,1]$ be a Whitney map. By the Hurewicz Theorem it suffices to prove that $\operatorname{dim}\left(\mu^{-1}(t)\right) \leq 1$ for each $t \in[0,1]$. This follows by Theorem 3.2 in case $\check{H}^{1}(X)=0$.

Suppose the above is true for all atriodic continua $X$ such that $\check{H}^{1}(X)$ has rank $\leq n$. Let $X$ be an atriodic continuum such that $\check{H}^{1}(X)$ has $\operatorname{rank} n+1$, let $\mu: C(X) \rightarrow[0,1]$ be a Whitney map, and $t \in[0,1]$.

Suppose first that for each proper subcontinuum $K$ of $X, \operatorname{rank}\left(\check{H}^{1}(K)\right) \leq n$. Let $\left\{U_{i}\right\}_{i=1}^{\infty}$ be a basis of open sets for $X$ such that $\lim _{i} \operatorname{diameter}\left(U_{i}\right)=0$. Then $\left\{X \backslash U_{i}\right\}_{i=1}^{\infty}$ is a sequence of closed proper subsets of $X$ such that

$$
C(X)=\bigcup_{i=1}^{\infty} C\left(X \backslash U_{i}\right) \cup\{X\}
$$

For each $i$ each component $K$ of $X \backslash U_{i}$ is atriodic and $\operatorname{rank}\left(\check{H}^{1}(K)\right) \leq n$. By induction $\operatorname{dim}\left(\mu^{-1}(t) \cap C(K) \leq 1\right.$. Hence, $\operatorname{dim}\left(\mu^{-1}(t) \cap C\left(X \backslash U_{i}\right)\right) \leq 1$ since the components of $\mu^{-1}(t) \cap C\left(X \backslash U_{i}\right)$ are exactly the sets $\mu^{-1}(t) \cap C(K)$, where $K$ is a component of $X \backslash U_{i}$. By the Sum Theorem for Dimension

$$
\operatorname{dim} \mu^{-1}(t)=\operatorname{dim}\left[\bigcup_{i=1}^{\infty}\left(\mu^{-1}(t) \cap C\left(X \backslash U_{i}\right)\right) \cup\{X\}\right]=1
$$

Let $f: X \rightarrow S^{1}$ be an essential map and let $A$ be a subcontinuum of $X$ such that $f$ is irreducibly essential on $A$. Let $E$ be a proper subcontinuum of $A$. To prove that $\operatorname{dim}\left(\mu^{-1}(t) \cap C(A)\right) \leq 1$ it suffices to prove by the above paragraph that $\check{H}^{1}(E)$ has rank $\leq n$. Just suppose that there exist linearly independent maps $g_{1}, \ldots, g_{n+1}$ : $E \rightarrow S^{1}$. By [6, Corollary 3.3] there exists for each $i=1, \ldots, n+1$ a continuous extension $\hat{g}_{i}: X \rightarrow S^{1}$ of $g_{i}$. Then $\left\{\hat{g}_{1}, \ldots, \hat{g}_{n+1}, f\right\}$ is linearly dependent. There exist integers $\alpha_{1}, \ldots, \alpha_{n+2}$ not all zero such that

$$
g=\hat{g}_{1}^{\alpha_{1}} \cdot \ldots \cdot \hat{g}_{n+1}^{\alpha_{n+1}} \cdot f^{\alpha_{n+2}}
$$

is inessential. Thus,

$$
g \mid E=g_{1}^{\alpha_{1}} \cdot \ldots \cdot g_{n+1}^{\alpha_{n+1}} \cdot(f \mid E)^{\alpha_{n+2}}
$$

is inessential. Also, $f \mid E$ is inessential so $g_{1}^{\alpha_{1}} \cdot \ldots \cdot g_{n+1}^{\alpha_{n+1}}$ is inessential. Since 
$\left\{g_{1}, \ldots, g_{n+1}\right\}$ is linearly independent $0=\alpha_{1}=\cdots=\alpha_{n+1}$ and, hence, $\alpha_{n+2} \neq$ 0 . Since $\breve{H}^{1}(X)$ is torsion free and $f$ is essential, $f^{\alpha_{n+2}}$ is essential. This is a contradiction.

Now, let $D$ be a continuum in $X \backslash A$. By [6] we may suppose that $f$ is an extension of $f \mid A$ to $X$ such that $f \mid D$ is inessential. By the same proof as above $\check{H}^{1}(D)$ has rank $\leq n$.

By [12, Lemma 8] there exist two points $p, q \in A$ such that if $B \in C(X)$ with $B \neq B \cap A \neq \varnothing$, then $\{p, q\} \cap B \neq \varnothing$. Hence,

$$
\begin{aligned}
\mu^{-1}(t)= & {\left[\mu^{-1}(t) \cap C(X \backslash A)\right] \cup\left\{B \in \mu^{-1}(t) \mid\{p, q\} \cap B \neq \varnothing\right\} } \\
& \cup\left(\mu^{-1}(t) \cap C(A)\right) .
\end{aligned}
$$

By Lemma $3.1\left\{B \in \mu^{-1}(t) \mid\{p, q\} \cap B \neq \varnothing\right\}$ is compact and contained in the union of two arcs and, so, is at most one-dimensional. For each positive integer $i$ let $X_{i}=X \backslash S\left(A, i^{-1}\right)$. Then,

$$
\mu^{-1}(t) \cap C(X \backslash A)=\mu^{-1}(t) \cap \bigcup_{i=l}^{\infty} C\left(X_{i}\right) .
$$

If $K$ is a component of $X_{i}$ then rank $\breve{H}^{1}(K) \leq n$ so $\operatorname{dim}\left(\mu^{-1}(t) \cap C(K)\right) \leq 1$. Each component of $\mu^{-1}(t) \cap C\left(X_{i}\right)$ has the form $\mu^{-1}(t) \cap C(K)$, where $K$ is a component of $X_{i}$. Hence, $\operatorname{dim}\left(\mu^{-1}(t) \cap C\left(X_{i}\right)\right) \leq 1$. By the Sum Theorem for Dimension $\operatorname{dim}\left(\mu^{-1}(t)\right) \leq 1$.

Problem. Let $X$ be an atriodic continuum embeddable in the plane. Is $\operatorname{dim}(C(X))$ $\leq 2$ ? Krasinkiewicz [10] has given a positive solution in case $X$ is hereditarily indecomposable.

\section{REFERENCES}

1. J. F. Davis, Atriodic acyclic continua and Class W, Proc. Amer. Math. Soc. 90 (1984), 477-482.

2. S. Eilenberg, Sur les transformation d'espaces metriques en circonference, Fund. Math. 24 (1935), 160-176.

3. J. Grispolakis and E. D. Tymchatyn, Continua which are images of weakly confluent mappings only. I, Houston J. Math. 5 (1979), 483-502.

4. __ Continua which are images of weakly confluent mappings only. II, Houston J. Math. 6 (1980), 375-387.

5. Soc. 74 (1979), 177-182.

6. __ On the Cech cohomology of continua with no n-ods, Houston J. Math. 11 (1985), 505-513.

7. W. Hurewicz and H. Wallman, Dimension theory, Princeton Univ. Press, Princeton, N.J., 1948 .

8. W. T. Ingram, $C$-sets and mappings of continua, Topology Proc. 7 (1982), 83-90.

9. J. L. Kelley, Hyperspaces of a continuum, Trans. Amer. Math. Soc. 52 (1942), 22-36.

10. J. Krasinkiewicz, On the hyperspaces of certain plane continua, Bull. Polish Acad. Sci. Math. 23 (1975), 981-983.

11. S. B. Nadler, Jr., Hyperspaces of sets, Dekker, New York, 1978.

12. V. C. Nall, Weak confluence and W-sets, Topology Proc. 8 (1983), 161-194.

13. L. G. Oversteegen and E. D. Tymchatyn, On atriodic tree-like continua, Proc. Amer. Math. Soc. 83 (1981), 201-204.

14. J. H. Roberts and N. E. Steenrod, Monotone transformations of 2-dimensional manifolds, Ann. of Math. (2) 39 (1939), 851-862.

15. J. T. Rogers, Jr., Weakly confluent maps and finitely-generated cohomology, Proc. Amer. Math. Soc. 78 (1980), 436-438. 
16. R. H. Sorgenfrey, Concerning triodic continua, Amer. J. Math. 66 (1944), 439-460.

17. E. D. Tymchatyn and B. O. Friberg, A problem of Martin concerning strongly convex metrics on $E^{3}$, Proc. Amer. Math. Soc. 43 (1974), 461-464.

18. L. Vietoris, Über den höheren Zusammenhang kompakten Räume und eine Klasse van zusammenhangstreuen Abbildungen, Math. Ann. 97 (1972), 454-472.

Department of Mathematics, Technology University of Crete, Chania, CRETE 73100, GREECE

Department of Mathematics, University of Saskatchewan, Saskatoon, SASKATCHEWAN S7N OW0, CANADA 